\title{
Interrogating the Molecular Basis for Substrate Recognition in Serotonin and Dopamine Transporters with High-Affinity Substrate-Based Bivalent Ligands
}

Jacob Andersen, ${ }^{* \dagger}$ Lucy Kate Ladefoged, ${ }^{\ddagger}$ Trine N. Bjerre Kristensen, ${ }^{\dagger}$ Lachlan Munro, ${ }^{\dagger}$ Julie Grouleff, ${ }^{\ddagger, \S}$ Nicolai Stuhr-Hansen, ${ }^{\dagger, \#}$ Anders S. Kristensen, ${ }^{\dagger}$ Birgit Schiøtt, ${ }^{\ddagger}$ and Kristian Strømgaard ${ }^{*}, \dagger$

'Department of Drug Design and Pharmacology, University of Copenhagen, DK-2100 Copenhagen, Denmark.

Interdisciplinary Nanoscience Center (iNANO), Department of Chemistry, Aarhus University, DK8000 Aarhus C, Denmark.

${ }^{\S}$ Present address: Ontario Institute for Cancer Research, MaRS Centre, Ontario M5G 0A3, Toronto, Canada.

\#Present address: Department of Chemistry, University of Copenhagen, DK-1871 Frederiksberg C, Denmark.

"E-mail: bunsejakse@gmail.com (J.A.); kristian.stromgaard@sund.ku.dk (K.S.).

Figure S1: Structure of compounds employed in the study

Figure S2: Structural alignment of hSERT and LeuT

Table S1: Results from IFD calculations

Table S2: Impact of hSERT mutations on 5HT uptake kinetics and potency of 5HT-PEG4-5HT

Table S3: Impact of hDAT mutations on DA uptake kinetics and potency of 5HT-PEG4-5HT S6

Table S4: Binding affinity of substrates and inhibitors at hSERT and hDAT mutants

Table S5: Functional activity of hSERT and hDAT mutants

Methods

Supporting references 


\section{Supporting figures and tables}

A

$$
\begin{aligned}
& \text { 5HT-PEG3-5HT }(n=1,1) \\
& \text { 5HT-PEG4-5HT }(n=2,2) \\
& \text { 5HT-PEG5-5HT }(n=3,3) \\
& \text { 5HT-PEG6-5HT }(n=4,4)
\end{aligned}
$$

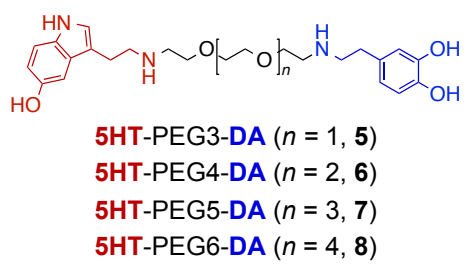

B

$$
\begin{aligned}
& 5 \mathrm{HT}-\mathrm{C}_{8} \mathrm{H}_{16}-5 \mathrm{HT}(n=1,13) \\
& 5 \mathrm{HT}-\mathrm{C}_{10} \mathrm{H}_{20}-5 \mathrm{HT}(n=2,14) \\
& 5 \mathrm{HT}-\mathrm{C}_{12} \mathrm{H}_{24}-5 \mathrm{HT}(n=3,15) \\
& 5 \mathrm{HT}-\mathrm{C}_{14} \mathrm{H}_{28}-5 \mathrm{HT}(n=4,16)
\end{aligned}
$$

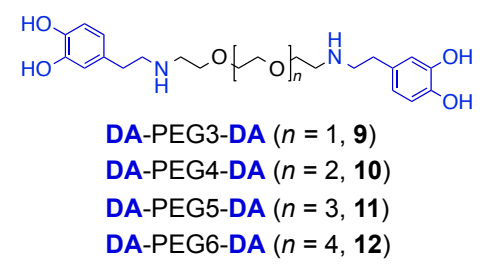

(n)

DA- $\mathrm{C}_{8} \mathrm{H}_{16}-\mathrm{DA}(n=1,21)$

DA- $\mathrm{C}_{10} \mathrm{H}_{20}$-DA $(n=2,22)$

DA- $\mathrm{C}_{12} \mathrm{H}_{24}$-DA $(n=3,23)$

DA- $\mathrm{C}_{14} \mathrm{H}_{28}$-DA $(n=4,24)$

\section{C}

$$
\text { 俯 }
$$

5HT-PEG2-Et ( $\left.n=1, \mathrm{R}=\mathrm{CH}_{2} \mathrm{CH}_{3}, 25\right)$ 5HT-PEG3-Et $\left(n=2, \mathrm{R}=\mathrm{CH}_{2} \mathrm{CH}_{3}, 26\right)$ 5HT-PEG4-Me ( $\left.n=3, \mathrm{R}=\mathrm{CH}_{3}, 27\right)$ 5HT-PEG5-Me $\left(n=4, \mathrm{R}=\mathrm{CH}_{3}, 28\right)$

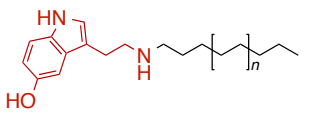

$5 \mathrm{HT}-\mathrm{C}_{8} \mathrm{H}_{17}(n=1,29)$ $5 \mathrm{HT}-\mathrm{C}_{10} \mathrm{H}_{21}(n=2,30)$ $5 \mathrm{HT}-\mathrm{C}_{12} \mathrm{H}_{25}(n=3,31)$ $5 \mathrm{HT}-\mathrm{C}_{14} \mathrm{H}_{29}(n=4,32)$

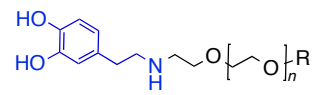

DA-PEG2-Et ( $\left.n=1, \mathrm{R}=\mathrm{CH}_{2} \mathrm{CH}_{3}, 33\right)$ DA-PEG3-Et $\left(n=2, \mathrm{R}=\mathrm{CH}_{2} \mathrm{CH}_{3}, \mathbf{3 4}\right)$ DA-PEG4-Me $\left(n=3, \mathrm{R}=\mathrm{CH}_{3}, 35\right)$ DA-PEG5-Me $\left(n=4, \mathrm{R}=\mathrm{CH}_{3}, 36\right)$

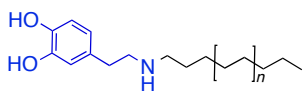

DA- $\mathrm{C}_{8} \mathrm{H}_{17}(n=1,37)$

DA- ${ }_{10} \mathrm{H}_{21}(n=2,38)$

DA- $\mathrm{C}_{12} \mathrm{H}_{25}(n=3,39)$

DA- $\mathrm{C}_{14} \mathrm{H}_{29}(n=4,40)$

Figure S1. Structure of compounds employed in the study which were synthesized as described previously. ${ }^{1}$ (A) Bivalent PEG-linked compounds (compound 1-12). (B) Bivalent alkyl-linked compounds (compound 1324). (C) Monomeric compounds attached to a PEG or an alkyl linker (compound 25-40). In order to mimic the bivalent PEG-linked structures, monomeric compounds should be attached to a PEG linker with a terminal ethyl group. Due to limited commercial availability of the required PEG-ethyl building blocks, compounds 27, 28, 35 and $\mathbf{3 6}$ are attached to a PEG linker with a terminal methyl group. 5HT moieties are shown in red and DA moieties are shown in blue. 

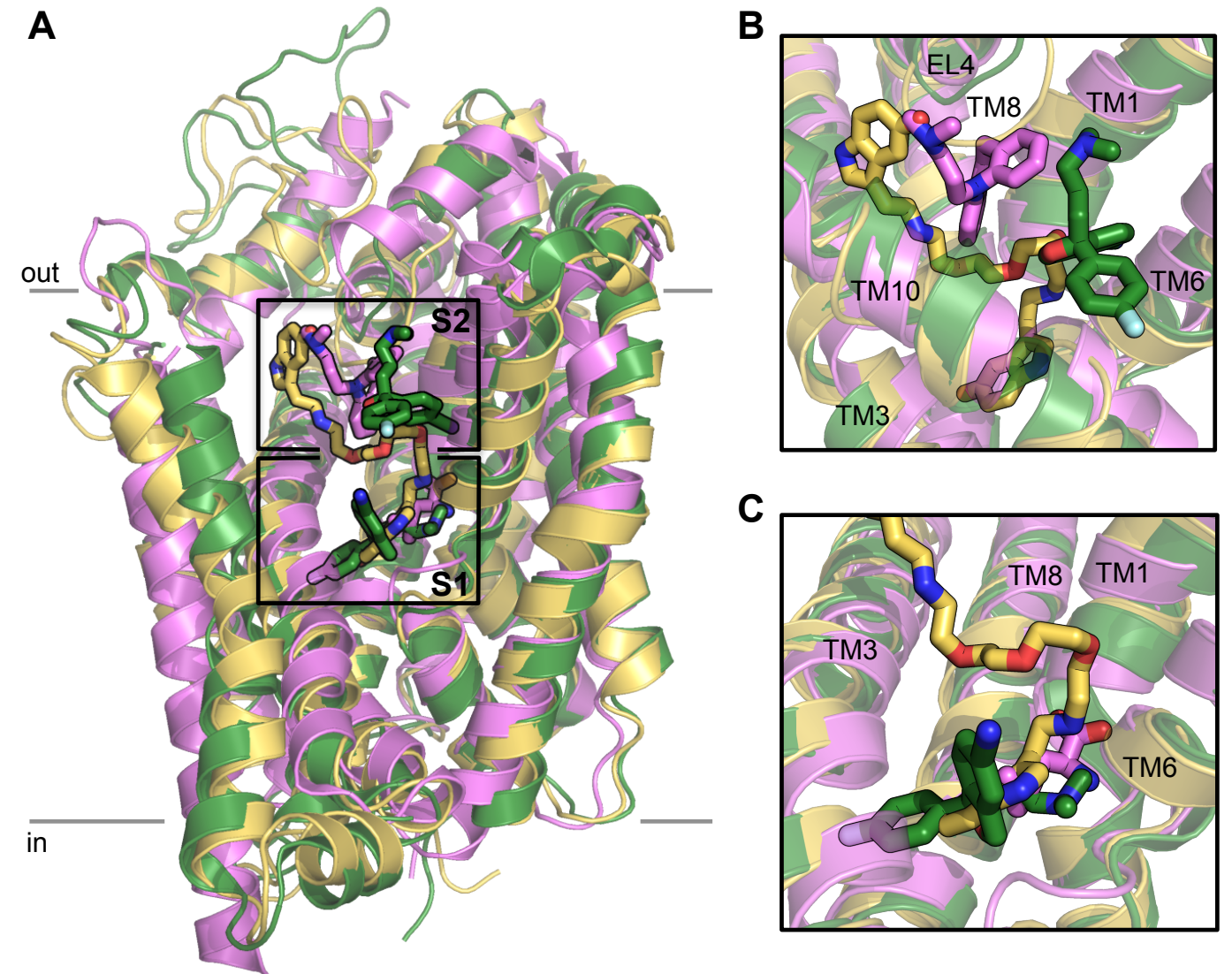

hSERT model with 5HT-PEG4-5HT (Cluster 2)

hSERT x-ray structure with escitalopram in S1 and S2 (PDB 5I73)

$\square$ LeuT x-ray structure with leucine in S1 and imipramine in S2 (PDB 2Q72)

Figure S2. Structural alignment of hSERT and LeuT. (A) Structural alignment of the binding model of 5HTPEG4-5HT in hSERT (Cluster 2; shown in yellow), hSERT crystal structure with escitalopram in the S1 and S2 sites (PDB 5I73; shown in green), and LeuT crystal structure with leucine in the S1 site and imipramine in the S2 site (PDB 2Q72; shown in magenta). TMs 10 and 11 have been removed for clarity, and ligands are shown as stick representations. (B) Close up view of ligand binding in the S2 site. 5HT-PEG4-5HT, escitalopram, and imipramine are shown as stick representations in yellow, green, and magenta, respectively. (C) Close up view of ligand binding in the S1 site. 5HT-PEG4-5HT, escitalopram, and leucine are shown as stick representations in yellow, green, and magenta, respectively. 
Table S1. Results from IFD calculations.

5HT-PEG4-5HT (2), 5HT-PEG4-DA (6) and DA-PEG4-DA (10) was docked into a homology model of hSERT WT. The number of poses in each binding cluster is listed, together with the average Glide Emodel and XP GScores (standard deviations shown in brackets). It is indicated which substrate moiety of the bivalent ligands that is bound within the central S1 site.

\begin{tabular}{lccccc}
\hline \multirow{2}{*}{ Compound } & \multirow{2}{*}{ Cluster } & \# Poses & Avg. Glide Emodel & Avg. XP Gscore & $\begin{array}{c}\text { Substrate moiety } \\
\text { in S1 site }\end{array}$ \\
\cline { 4 - 5 } 5HT-PEG4-5HT (2) & 1 & 3 & $-118.1(0.4)$ & $-11.1(1.0)$ & $5 \mathrm{HT}$ \\
& 2 & 3 & $-108.2(6.9)$ & $-11.7(0.4)$ & $5 \mathrm{HT}$ \\
& 3 & 1 & $-121.3(-)$ & $-12.2(-)$ & $5 \mathrm{HT}$ \\
& & & & & \\
5HT-PEG4-DA (6) & 1 & 1 & $-104.4(-)$ & $-10.7(-)$ & DA \\
& 2 & 1 & $-120.9(-)$ & $-14.3(-)$ & DA \\
& 3 & 7 & $-109.7(4.3)$ & $-13.1(0.5)$ & DA \\
& 4 & 1 & $-81.5(-)$ & $-11.3(-)$ & $5 \mathrm{HT}$ \\
& 5 & 6 & $-97.6(17.0)$ & $-11.9(1.0)$ & $5 \mathrm{HT}$ \\
DA-PEG4-DA (10) & 1 & 8 & $-97.7(7.8)$ & $-12.3(0.4)$ & DA \\
& 2 & 1 & $-97.8(-)$ & $-11.7(-)$ & DA \\
& 3 & 3 & $-105.8(7.0)$ & $-13.1(0.8)$ & DA \\
\hline
\end{tabular}


Table S2. Impact of hSERT mutations on $\left[{ }^{3} \mathrm{H}\right] 5 \mathrm{HT}$ uptake kinetics and potency of 5HT-PEG4-5HT. Uptake activity, 5HT $\mathrm{K}_{\mathrm{m}}$ and 5HT-PEG4-5HT $\mathrm{K}_{\mathrm{i}}$ values were determined in $\left[{ }^{3} \mathrm{H}\right] 5 \mathrm{HT}$ uptake assays. Results are presented as mean \pm SEM from $n$ independent experiments each performed in triplicate. Uptake activity was determined after 5 min uptake with $50-100 \mathrm{nM}\left[{ }^{3} \mathrm{H}\right] 5 \mathrm{HT}$ and is presented relative to hSERT WT activity from paired experiments. The fold-changes in $\mathrm{K}_{\mathrm{i}}$ values were calculated as $-\mathrm{K}_{\mathrm{i}}($ mutant $) / \mathrm{K}_{\mathrm{i}}(\mathrm{WT})$ or $\mathrm{K}_{\mathrm{i}}(\mathrm{WT}) / \mathrm{K}_{\mathrm{i}}$ (mutant) for mutants decreasing or increasing the potency of 5HT-PEG4-5HT, respectively. Asterisk $\left({ }^{*}\right)$ denotes significantly different $\mathrm{K}_{\mathrm{i}}$ value compared to hSERT WT (Student's $t$-test; $\mathrm{p}<0.05$ ).

\begin{tabular}{|c|c|c|c|c|c|c|c|c|}
\hline \multirow{3}{*}{$\begin{array}{l}\text { Mutant } \\
\text { hSERT WT }\end{array}$} & \multicolumn{4}{|c|}{$5 \mathrm{HT} \mathrm{K}_{\mathrm{m}}$} & \multirow{2}{*}{$\begin{array}{l}\text { Uptake activity } \\
\% \text { of } h S E R T W T\end{array}$} & \multicolumn{2}{|c|}{ 5HT-PEG4-5HT K ${ }_{\mathrm{i}}$} & \multirow{2}{*}{$\begin{array}{c}\text { Fold-change } \\
\text { in } K_{i} \text {-value }\end{array}$} \\
\hline & \multicolumn{3}{|c|}{$\mu M$} & \multirow{2}{*}{$\begin{array}{l}n \\
9\end{array}$} & & $n M$ & \multirow{2}{*}{$\begin{array}{l}n \\
44\end{array}$} & \\
\hline & 1.90 & \pm & 0.20 & & 100 & $13.4 \pm 1.2$ & & \\
\hline Y95A & 0.22 & \pm & $0.04^{\mathrm{a}}$ & 3 & $43 \pm 5$ & $24.2 \pm 4.4^{*}$ & 6 & -1.8 \\
\hline Y95F & 0.35 & \pm & $0.09^{a}$ & 4 & $66 \pm 12$ & $5.3 \pm 2.0$ & 4 & 2.5 \\
\hline Y95W & 2.16 & \pm & $0.25^{\mathrm{b}}$ & 4 & $26 \pm 3$ & $7.0 \pm 2.0$ & 4 & 1.9 \\
\hline D98E & 0.22 & \pm & $0.06^{a}$ & 3 & $46 \pm 11$ & $5.6 \pm 1.7^{*}$ & 5 & 2.4 \\
\hline W103A & 0.96 & \pm & $0.09^{b}$ & 5 & $13 \pm 2$ & $10.6 \pm 3.6$ & 5 & 1.3 \\
\hline A169D & 0.62 & \pm & $0.06^{a}$ & 3 & $91 \pm 9$ & $4.5 \pm 1.3^{*}$ & 4 & 3.0 \\
\hline A169I & 3.62 & \pm & $0.94^{a}$ & 5 & $51 \pm 4$ & $57.8 \pm 6.5^{*}$ & 8 & -4.3 \\
\hline A169S & 0.85 & \pm & $0.35^{a}$ & 5 & $94 \pm 10$ & $17.2 \pm 2.6$ & 5 & -1.3 \\
\hline F170L & 0.65 & \pm & $0.08^{a}$ & 3 & $91 \pm 8$ & $8.9 \pm 2.3$ & 4 & 1.5 \\
\hline $\mathrm{I} 172 \mathrm{~F}$ & 1.16 & \pm & $0.20^{\mathrm{a}}$ & 3 & $29 \pm 3$ & $19.2 \pm 2.0$ & 3 & -1.4 \\
\hline I172M & 0.77 & \pm & $0.05^{c}$ & 4 & $87 \pm 6$ & $10.0 \pm 1.9$ & 6 & 1.3 \\
\hline I172Q & 3.12 & \pm & $0.72^{\mathrm{a}}$ & 3 & $21 \pm 2$ & $20.1 \pm 3.2$ & 3 & -1.5 \\
\hline I172Y & 4.25 & \pm & $0.27^{d}$ & 6 & $19 \pm 1$ & $22.2 \pm 4.3$ & 3 & -1.7 \\
\hline A173G & 0.26 & \pm & $0.07^{a}$ & 3 & $42 \pm 7$ & $2.6 \pm 1.0^{*}$ & 4 & 5.1 \\
\hline A173I & 1.17 & \pm & $0.34^{a}$ & 5 & $79 \pm 12$ & $7.3 \pm 2.6$ & 4 & 1.8 \\
\hline Y175F & 0.24 & \pm & $0.08^{a}$ & 3 & $58 \pm 4$ & $7.8 \pm 0.3$ & 4 & 1.7 \\
\hline Y176F & 0.88 & \pm & $0.04^{a}$ & 3 & $80 \pm 14$ & $6.1 \pm 2.5$ & 4 & 2.2 \\
\hline N177A & 7.65 & \pm & $0.42^{\mathrm{a}}$ & 3 & $73 \pm 5$ & $279 \pm 29^{*}$ & 8 & -20.8 \\
\hline N177C & 5.71 & \pm & 0.26 & 3 & $126 \pm 16$ & $158 \pm 16^{\star}$ & 5 & -11.8 \\
\hline N177E & 1.55 & \pm & $0.14^{\mathrm{a}}$ & 3 & $102 \pm 11$ & $22.2 \pm 2.4^{*}$ & 6 & -1.7 \\
\hline N177G & 11.9 & \pm & 1.42 & 3 & $73 \pm 7$ & $310 \pm 36^{*}$ & 6 & -23.1 \\
\hline N177S & 4.25 & \pm & $0.12^{\mathrm{a}}$ & 3 & $90 \pm 10$ & $95.8 \pm 9.8^{*}$ & 10 & -7.2 \\
\hline I179C & 0.53 & \pm & $0.11^{\mathrm{b}}$ & 3 & $14 \pm 2$ & $2.9 \pm 0.7^{*}$ & 5 & 4.6 \\
\hline W182A & 2.22 & \pm & $0.26^{b}$ & 5 & $97 \pm 17$ & $13.7 \pm 2.5$ & 4 & 1.0 \\
\hline Y232A & 0.19 & \pm & $0.02^{b}$ & 5 & $28 \pm 5$ & $15.8 \pm 5.8$ & 4 & -1.2 \\
\hline Y232F & 1.33 & \pm & $0.18^{b}$ & 5 & $63 \pm 13$ & $13.5 \pm 3.7$ & 4 & 1.0 \\
\hline F335A & 0.33 & \pm & $0.11^{\mathrm{a}}$ & 3 & $31 \pm 5$ & $6.7 \pm 0.5$ & 5 & 2.0 \\
\hline F335I & 0.85 & \pm & $0.20^{\mathrm{a}}$ & 3 & $84 \pm 9$ & $13.8 \pm 2.3$ & 5 & 1.0 \\
\hline F335W & 0.06 & \pm & $0.01^{a}$ & 3 & $23 \pm 5$ & $9.7 \pm 1.7$ & 4 & 1.4 \\
\hline F341Y & 0.08 & \pm & $0.02^{\mathrm{a}}$ & 3 & $55 \pm 8$ & $19.0 \pm 2.7$ & 6 & -1.4 \\
\hline F341W & 0.99 & \pm & $0.14^{d}$ & 6 & $49 \pm 8$ & $17.9 \pm 1.8$ & 3 & -1.3 \\
\hline V343N & 0.24 & \pm & $0.01^{\mathrm{a}}$ & 3 & $17 \pm 3$ & $5.6 \pm 1.9$ & 4 & 2.4 \\
\hline D400K & 0.12 & \pm & $0.03^{\mathrm{e}}$ & 3 & $14 \pm 2$ & $5.1 \pm 1.1^{*}$ & 5 & 2.6 \\
\hline P403A & 1.49 & \pm & $0.08^{b}$ & 5 & $64 \pm 8$ & $8.7 \pm 2.8$ & 4 & 1.5 \\
\hline P403E & 0.30 & \pm & $0.04^{b}$ & 5 & $35 \pm 12$ & $7.5 \pm 2.2$ & 3 & 1.8 \\
\hline P403I & 0.95 & \pm & 0.13 & 3 & $49 \pm 13$ & $9.4 \pm 1.8$ & 3 & 1.4 \\
\hline P403S & 0.67 & \pm & 0.14 & 5 & $43 \pm 15$ & $6.6 \pm 1.9^{*}$ & 10 & 2.0 \\
\hline S438A & 0.46 & \pm & $0.09^{a}$ & 3 & $63 \pm 26$ & $6.1 \pm 1.3$ & 3 & 2.2 \\
\hline S438T & 0.14 & \pm & $0.02^{a}$ & 3 & $35 \pm 2$ & $13.4 \pm 2.0$ & 6 & 1.0 \\
\hline
\end{tabular}

Table S2 continued on next page. 


\begin{tabular}{|c|c|c|c|c|c|c|c|c|}
\hline \multirow{3}{*}{$\begin{array}{l}\text { Mutant } \\
\text { S438A }\end{array}$} & \multicolumn{4}{|c|}{$5 \mathrm{HT} \mathrm{K}_{\mathrm{m}}$} & \multirow{2}{*}{$\begin{array}{l}\text { Uptake activity } \\
\text { \% of } h S E R T W T\end{array}$} & \multicolumn{2}{|c|}{ 5HT-PEG4-5HT $\mathrm{K}_{\mathrm{i}}$} & \multirow{3}{*}{$\begin{array}{c}\text { Fold-change } \\
\text { in } \mathbf{K}_{\mathrm{i}} \text {-value } \\
2.2\end{array}$} \\
\hline & \multicolumn{3}{|c|}{$\mu M$} & \multirow{2}{*}{$\begin{array}{l}n \\
3\end{array}$} & & $n M$ & $n$ & \\
\hline & 0.46 & \pm & $0.09^{a}$ & & $63 \pm 26$ & $6.1 \pm 1.3$ & 3 & \\
\hline $\mathrm{S} 438 \mathrm{~T}$ & 0.14 & \pm & $0.02^{\mathrm{a}}$ & 3 & $35 \pm 2$ & $13.4 \pm 2.0$ & 6 & 1.0 \\
\hline T439A & 8.21 & \pm & $0.82^{a}$ & 3 & $18 \pm 2$ & $31.7 \pm 8.7^{*}$ & 4 & -2.4 \\
\hline T439C & 7.84 & \pm & 1.01 & 5 & $65 \pm 9$ & $62.1 \pm 12^{*}$ & 5 & -4.6 \\
\hline T439S & 0.75 & \pm & $0.10^{\mathrm{a}}$ & 3 & $66 \pm 4$ & $23.9 \pm 3.7^{*}$ & 4 & -1.8 \\
\hline T439V & 9.35 & \pm & $1.26^{a}$ & 5 & $27 \pm 3$ & $151 \pm 16^{*}$ & 9 & -11.3 \\
\hline A486E & 0.51 & \pm & $0.09^{b}$ & 5 & $39 \pm 4$ & $5.3 \pm 1.6^{*}$ & 6 & 2.5 \\
\hline A486I & 0.46 & \pm & $0.06^{b}$ & 5 & $11 \pm 1$ & $4.0 \pm 1.9^{*}$ & 4 & 3.4 \\
\hline V489F & 0.63 & \pm & $0.43^{\mathrm{e}}$ & 3 & $73 \pm 12$ & $2.9 \pm 0.9^{*}$ & 5 & 4.6 \\
\hline K490D & 0.16 & \pm & $0.02^{\mathrm{e}}$ & 4 & $41 \pm 8$ & $2.5 \pm 0.8^{*}$ & 8 & 5.3 \\
\hline K490E & 1.29 & \pm & 0.12 & 3 & $103 \pm 28$ & $11.4 \pm 0.8$ & 3 & 1.2 \\
\hline K490F & 0.17 & \pm & $0.06^{\mathrm{e}}$ & 3 & $95 \pm 28$ & $6.5 \pm 0.8$ & 3 & 2.1 \\
\hline K490Q & 1.13 & \pm & 0.18 & 3 & $121 \pm 38$ & $11.5 \pm 1.8$ & 3 & 1.2 \\
\hline K490T & 0.27 & \pm & $0.09^{e}$ & 4 & $33 \pm 3$ & $3.1 \pm 0.8^{*}$ & 6 & 4.4 \\
\hline E493A & 1.50 & \pm & $0.11^{\mathrm{b}}$ & 5 & $34 \pm 6$ & $8.9 \pm 0.3$ & 4 & 1.5 \\
\hline N177C-T439C & 14.3 & \pm & 1.06 & 3 & $95 \pm 10$ & $114 \pm 6.1^{*}$ & 5 & -8.5 \\
\hline N177A-T439V & 35.2 & \pm & 4.17 & 3 & $8 \pm 2$ & $571 \pm 101^{*}$ & 5 & -42.6 \\
\hline P403S-K490D & 0.97 & \pm & 0.09 & 3 & $56 \pm 13$ & $10.1 \pm 1.1$ & 5 & 1.3 \\
\hline C109A-S404C & 1.70 & \pm & 0.04 & 3 & $57 \pm 11$ & $11.6 \pm 1.5$ & 6 & 1.2 \\
\hline C109A-S404C-N177A & 11.0 & \pm & 2.16 & 4 & $35 \pm 3$ & $228 \pm 45^{\star}$ & 4 & -17.0 \\
\hline C109A-S404C-T439V & 11.0 & \pm & 4.97 & 2 & $9 \pm 1$ & $110 \pm 28^{*}$ & 4 & -8.2 \\
\hline
\end{tabular}

${ }^{a}$ Value taken from Andersen et al. (2010) J. Biol. Chem. 285, 2051-2063.

${ }^{b}$ Value taken from Andersen et al. (2014) Mol. Pharmacol. 85, 703-714.

${ }^{c}$ Value taken from Sørensen et al. (2012) J. Biol. Chem. 287, 43694-43707.

${ }^{d}$ Value taken from Andersen et al. (2015) ACS Chem. Neurosci. 6, 1892-1900.

${ }^{e}$ Value taken from Andersen et al. (2009) J. Biol. Chem. 284, 10276-10284.

Table S3. Impact of hDAT mutations on $\left[{ }^{3} \mathrm{H}\right] \mathrm{DA}$ uptake kinetics and potency of 5HT-PEG4-5HT. Uptake activity, DA $\mathrm{K}_{\mathrm{m}}$ and 5HT-PEG4-5HT $\mathrm{K}_{\mathrm{i}}$ values were determined in $\left[{ }^{3} \mathrm{H}\right] \mathrm{DA}$ uptake assays. Results are presented as mean \pm SEM from $n$ independent experiments each performed in triplicate. Uptake activity was determined after 5 min uptake with $50 \mathrm{nM}\left[{ }^{3} \mathrm{H}\right] \mathrm{DA}$ and is presented relative to hDAT WT activity from paired experiments. The fold-changes in $\mathrm{K}_{\mathrm{i}}$ values were calculated as $-\mathrm{K}_{\mathrm{i}}($ mutant $) / \mathrm{K}_{\mathrm{i}}(\mathrm{WT})$. Asterisk (*) denotes significantly different $\mathrm{K}_{\mathrm{i}}$ value compared to hDAT WT (Student's $t$-test; $\mathrm{p}<0.05$ ).

\begin{tabular}{|c|c|c|c|c|c|c|c|}
\hline \multirow{2}{*}{ Mutant } & \multicolumn{3}{|c|}{$\mathrm{DA} \mathrm{K}_{\mathrm{m}}$} & \multirow{3}{*}{$\begin{array}{c}\text { Uptake activity } \\
\% \text { of } h D A T W T \\
100\end{array}$} & \multicolumn{2}{|c|}{ 5HT-PEG4-5HT K ${ }_{i}$} & \multirow{2}{*}{$\begin{array}{l}\text { Fold-change } \\
\text { in } K_{\mathrm{i}} \text {-value }\end{array}$} \\
\hline & & $\mu M$ & $n$ & & $n M$ & $n$ & \\
\hline hDAT WT & 1.2 & \pm 0.1 & 3 & & $4,694 \pm 519$ & 10 & \\
\hline N157A & 1.4 & 0.4 & 3 & $30 \pm 3$ & $12,517 \pm 2,873^{*}$ & 7 & -2.7 \\
\hline A423T & 1.8 & 0.6 & 3 & $103 \pm 14$ & $4,989 \pm 1,516$ & 6 & -1.1 \\
\hline
\end{tabular}


Table S4. Binding affinity of substrates and inhibitors at hSERT (top) and hDAT (bottom) mutants. The binding affinity $\left(\mathrm{K}_{\mathrm{i}}\right)$ was determined in a $\left[{ }^{125} \mathrm{I}\right] \mathrm{RTI}-55$ competition binding assay. Data are presented as mean \pm SEM from $n$ independent experiments each performed in dublicate. Asterisk $(*)$ denotes significantly different $\mathrm{K}_{\mathrm{i}}$ value compared to the parent WT transporter (Student's $t$-test; $\mathrm{p}<0.05$ ).

\begin{tabular}{|c|c|c|c|c|c|c|c|c|}
\hline \multirow{3}{*}{$\begin{array}{l}\text { Compound } \\
\text { RTI-55 }\end{array}$} & \multicolumn{2}{|l|}{ hSERT WT } & \multicolumn{2}{|l|}{ SERT-(DAT S1) $^{b}$} & \multicolumn{4}{|c|}{ SERT-(DAT S2) $^{c}$} \\
\hline & $n M$ & \multirow{2}{*}{$\begin{array}{l}n \\
9\end{array}$} & $n M$ & \multirow{2}{*}{$\begin{array}{l}n \\
5\end{array}$} & \multicolumn{3}{|c|}{$n M$} & \multirow{2}{*}{$\frac{n}{4}$} \\
\hline & $3.6 \pm 1.4$ & & $2.9 \pm 1.3$ & & 16.1 & \pm & $1.6^{*}$ & \\
\hline $5 \mathrm{HT}$ & $2,426 \pm 456$ & 16 & $2,028 \pm 997$ & 7 & 27.6 & \pm & $10.7^{*}$ & 3 \\
\hline DA & $587,210 \pm 80,744$ & 6 & $515,562 \pm 108,725$ & 4 & 53,153 & \pm & $14170^{*}$ & 4 \\
\hline 5HT-PEG4-5HT (2) & $0.64 \pm 0.1$ & 9 & $0.39 \pm 0.07$ & 5 & 2.04 & \pm & $0.83^{*}$ & 5 \\
\hline 5HT-PEG4-DA (6) & $7.9 \pm 1.4$ & 4 & $7.6 \pm 0.6$ & 3 & 123 & \pm & $38^{*}$ & 3 \\
\hline DA-PEG4-DA (10) & $1,056 \pm 131$ & 5 & $518 \pm 126^{*}$ & 4 & 8,678 & \pm & $2589^{*}$ & 5 \\
\hline \multirow{2}{*}{ Compound } & \multicolumn{2}{|l|}{ hDAT WT } & \multicolumn{2}{|l|}{ DAT-(SERT S1) $^{d}$} & \multicolumn{4}{|c|}{ DAT-(SERT S2) ${ }^{e}$} \\
\hline & $n M$ & $n$ & $n M$ & $n$ & \multicolumn{3}{|c|}{$n M$} & $n$ \\
\hline RTI-55 & $0.86 \pm 0.13$ & 8 & $0.82 \pm 0.09$ & 3 & 1.8 & \pm & $0.2^{*}$ & 3 \\
\hline $5 \mathrm{HT}$ & $143,412 \pm 27,423$ & 6 & $126,550 \pm 21,154$ & 3 & 244,034 & \pm & 101,488 & 3 \\
\hline DA & $4,877 \pm 539$ & 5 & $5,353 \pm 797$ & 5 & 12,324 & \pm & 4,544 & 5 \\
\hline 5HT-PEG4-5HT (2) & $1,676 \pm 128$ & 7 & $1,472 \pm 208$ & 4 & 24,949 & \pm & $4853^{*}$ & 5 \\
\hline
\end{tabular}

${ }^{a} \mathrm{~K}_{\mathrm{D}}$ values from a $\left[{ }^{125} \mathrm{I}\right] \mathrm{RTI}-55$ saturation binding assay.

${ }^{b}$ SERT-(DAT S1) = hSERT-Y95F-G100A-I165V-C166I-A169S-F170L-I172V-A173G-S174F-Y175F-I333VF334C-T439A-A441G-L443M-G445S-C473T-T497A-V501I.

${ }^{c}$ SERT-(DAT S2) = hSERT-G100A-I108L-Q111K-Y175F-D400K-A401D-S404G-L406I-A486I-V489FK490T-E493D-T497A.

${ }^{d}$ DAT-(SERT S1) = hDAT-F76Y-A81G-V145I-I146C-S149A-L150F-V152I-G153A-F154S-F155Y-V318IC319F-A423T-G425A-M427L-S429G-T456C-A480T-I484V.

${ }^{e}$ DAT-(SERT S2) = A81G-L89I-K92Q-F155Y-K384D-D385A-G388S-I390L-I469A-F472V-T473K-D476EA480T.

Table S5. Functional activity of hSERT and hDAT mutants.

The uptake activity of hSERT and hDAT mutants relative to WT transporters was determined from paired uptake experiments. The uptake activity is expressed as percentage of hSERT WT (for $\left.{ }^{3} \mathrm{H}\right] 5 \mathrm{HT}$ uptake) or hDAT WT (for $\left[{ }^{3} \mathrm{H}\right] \mathrm{DA}$ uptake) after $5 \mathrm{~min}$ incubation with $100 \mathrm{nM}\left[{ }^{3} \mathrm{H}\right]$ substrate. Data are presented as mean \pm SEM from 4 independent experiments each performed in triplicate. N.F. = non-functional (defined as $<2 \%$ uptake activity relative to WT transporter).

\begin{tabular}{lccc}
\hline & {$\left[{ }^{3} \mathrm{H}\right] 5 \mathrm{HT}$ uptake } & & {$\left[{ }^{3} \mathrm{H}\right] \mathrm{DA}$ uptake } \\
\cline { 2 - 2 } hSERT WT & $\%$ of $h$ SERT WT & $\%$ of $h D A T W T$ \\
SERT-(DAT S1) & 100 & $3 \pm 1$ \\
SERT-(DAT S2) & $20 \pm 1$ & N.F. \\
\hline hDAT WT & $6 \pm 2$ & N.F. \\
DAT-(SERT S1) & N.F. & 100 \\
DAT-(SERT S2) & $5 \pm 2$ & N.F. \\
\hline
\end{tabular}




\section{Methods}

Materials. Dulbecco's modified Eagle's medium (DMEM), fetal bovine serum, trypsin, and penicillin-streptomycin was purchased from Invitrogen. Cell culture dishes were from Sarstedt (Nümbrecht, Germany), and 96-well plates from Nunc (Roskilde, Denmark). [ $\left.{ }^{3} \mathrm{H}\right] 5 \mathrm{HT}$ (20-30 $\mathrm{Ci} / \mathrm{mmol}),\left[{ }^{3} \mathrm{H}\right] \mathrm{DA}(60-80 \mathrm{Ci} / \mathrm{mmol}),\left[{ }^{125} \mathrm{I}\right] \mathrm{RTI}-55$ (2200 Ci/mmol), MicroScint-0 and MicroScint-20 were purchased from PerkinElmer (Waltham, MA, USA). Unlabelled RTI-55 was purchased from ABX (Radeberg, Germany). 2-Aminoethyl methane thiosulfonate hydrobromide (MTSEA) was purchased from Toronto Research Chemicals (Toronto, Ontario, Canada). Compounds $\mathbf{1}$ - $\mathbf{4 0}$ was synthesized as described previously. ${ }^{1}$ Compounds $\mathbf{1}-\mathbf{4 0}$ displayed only negligible decomposition when stored in sealed glass-jars for weeks at ambient conditions, and they proved high stability for several months when stored at $-20^{\circ} \mathrm{C}$ in $10 \mathrm{mM}$ stock solutions (as determined by NMR and UPLC analyses, respectively).

Molecular biology. As expression vectors, pcDNA3.1 or pCI-IRES-neo (a modified version of pCIneo) containing hSERT or hDAT cDNA were used. Generation of point mutations in hSERT and hDAT was performed by site-directed mutagenesis using the QuickChange site-directed mutagenesis kit (Stratagene, Carlsbad, CA, USA). Multiple mutants were generated by introducing one or more mutations into existing mutants by site-directed mutagenesis using the QuickChange mutagenesis kit. For construction of the DAT-(SERT S1) and DAT-(SERT S2) mutants, synthetic cDNA encoding the entire hDAT sequence with 19 hDAT-to-hSERT mutations in the S1 site [for DAT-(SERT S1)] or 13 hDAT-to-hSERT mutations in the S2 site [for DAT-(SERT S2)] was purchased from GeneArt. The synthetic DNA was digested with $X b a \mathrm{I}$ and $\mathrm{XhoI}$ and then ligated into $\mathrm{Xba \textrm {I } / X h o I}$ digested pCI-IRESneo vector using the Rapid DNA ligation kit (Roche, Mannheim, Germany). The sequence integrity of all mutants was verified by DNA sequencing (GATC Biotech, Cologne, Germany).

Cell culturing and transfection. COS-7 cells (American Type Culture Collection, Manassas, VA, USA) were cultured in growth medium (DMEM supplemented with $10 \%$ fetal bovine serum, 100 $\mathrm{U} / \mathrm{mL}$ penicillin, and $100 \mu \mathrm{g} / \mathrm{mL}$ streptomycin) at $37^{\circ} \mathrm{C}$ in a humidified $5 \% \mathrm{CO}_{2}$ environment. Cells were transfected using TransIT LT-1 DNA transfection reagent (Mirus, Madison, WI, USA) by following the procedure supplied by the manufacturer, and dispensed into either poly-D-lysine coated white 96-well plates (for uptake assays) or $150 \mathrm{~mm}$ tissue culture Petri plates (for membrane preparations).

$\left[{ }^{3}\right.$ H]5HT and $\left[{ }^{3}\right.$ H]DA uptake assays. Uptake assays were performed $40-48 \mathrm{~h}$ after transfection, and all washing steps were performed using an automated plate washer (ELx50, Bio-Tek Instruments Inc., Winooski, VT, USA). Before uptake experiments, cells were washed twice with PBSCM buffer (137 $\mathrm{mM} \mathrm{NaCl}, 2.7 \mathrm{mM} \mathrm{KCl}, 4.3 \mathrm{mM} \mathrm{Na}_{2} \mathrm{HPO}_{4}, 1.4 \mathrm{mM} \mathrm{KH}_{2} \mathrm{PO}_{4}, 0.1 \mathrm{mM} \mathrm{CaCl}_{2}$ and $0.5 \mathrm{mM} \mathrm{MgCl}_{2}$ ) and allowed to equilibrate for 20 to $30 \mathrm{~min}$ in $50 \mu \mathrm{L}$ of PBSCM per well. In all hDAT assays, the PBSCM buffer was supplemented with $1 \mathrm{mM}$ ascorbic acid and $1 \mu \mathrm{M}$ of the catechol-O-methyltransferase inhibitor Ro 41-0960. For transport inhibition experiments ( $\mathrm{IC}_{50}$ determinations), cells were preincubated with $40 \mu \mathrm{L}$ of PBSCM per well with no or increasing concentrations of inhibitor for 30 min before addition of $10 \mu \mathrm{L}$ of PBSCM containing $\left[{ }^{3} \mathrm{H}\right] 5 \mathrm{HT}$ (for hSERT assays) or $\left[{ }^{3} \mathrm{H}\right] \mathrm{DA}$ (for hDAT assays) giving a final substrate concentration of 50-100 $\mathrm{nM}$. Uptake was allowed to proceed for $5 \mathrm{~min}$ at $20^{\circ} \mathrm{C}$ before uptake was terminated by rapid washing three times with PBSCM. For determination of substrate $\mathrm{K}_{\mathrm{m}}$ values, cells were incubated with increasing concentrations of unlabelled 5HT (for hSERT assays) or DA (for hDAT assays) in PBSCM containing 50-100 nM $\left[{ }^{3} \mathrm{H}\right] 5 \mathrm{HT}$ (for hSERT assays) or $\left[{ }^{3} \mathrm{H}\right] \mathrm{DA}$ (for hDAT assays) for $5 \mathrm{~min}$ at $20^{\circ} \mathrm{C}$. Where indicated, the $\mathrm{K}_{\mathrm{m}}$ values were also determined in uptake saturation assays for selected mutants. Here, cells were incubated with increasing concentrations of $\left[{ }^{3} \mathrm{H}\right] 5 \mathrm{HT}$ (for hSERT assays) or $\left[{ }^{3} \mathrm{H}\right] \mathrm{DA}$ (for hDAT assays) diluted 1:30 (for hSERT assays) or 1:15 (for hDAT assays) with unlabelled substrate for 5 min at $20^{\circ} \mathrm{C}$. For all transport assays, the amount of accumulated radioligand was determined by solubilising cells in MicroScint-20 followed by counting of plates in a Packard TopCounter. Nonspecific uptake was determined as uptake in non-transfected cells, and total uptake was determined in 
the presence of PBSCM buffer alone. Assays were carried out in triplicate and repeated at least three times.

Measurement of S404C accessibility to MTSEA. COS-7 cells were transiently transfected with C109A-S404C and plated into 96-well plates as described above. $40-48 \mathrm{~h}$ after transfection, the cells were washed and incubated with increasing concentrations of MTSEA in the absence (control) or presence of a fixed concentration of inhibitor for $10 \mathrm{~min}$ at $20^{\circ} \mathrm{C}$. The cells were washed twice and incubated for $30 \mathrm{~min}$ in PBSCM to release bound inhibitor. Incubation of cells in PBSCM had no effect on MTSEA reaction (data not shown). As control, we included cells expressing C109A-S404C that were incubated with inhibitor alone. The residual uptake activity was determined by measuring uptake of $50 \mathrm{nM}\left[{ }^{3} \mathrm{H}\right] 5 \mathrm{HT}$ for $5 \mathrm{~min}$ at $20^{\circ} \mathrm{C}$. To examine if N177A or T439V affected accessibility of S404C, COS-7 cells expressing C109A-S404C, C109A-S404C-N177A or C109A-S404C-T439V were assayed in parallel and incubated with MTSEA for $10 \mathrm{~min}$. The cells were washed twice, and residual uptake activity was determined by measuring uptake of $100 \mathrm{nM}\left[{ }^{3} \mathrm{H}\right] 5 \mathrm{HT}$ for $5 \mathrm{~min}$ at $20^{\circ} \mathrm{C}$. Cells were washed and accumulated $\left[{ }^{3} \mathrm{H}\right] 5 \mathrm{HT}$ was quantified as described above.

Cell membrane preparations and binding assays. Cell membranes were prepared $40-48 \mathrm{~h}$ after transfection. Transiently transfected COS-7 cells growing in $150 \mathrm{~mm}$ tissue culture Petri plates were washed with PBS supplemented with $1 \mathrm{mM}$ EDTA to detach from the plate. Cell suspension was centrifuged $(700 \times \mathrm{g})$ for $5 \mathrm{~min}$, and the cell pellet was suspended in cold $\mathrm{H}_{2} \mathrm{O}$ and frozen at $-20^{\circ} \mathrm{C}$ for at least $1 \mathrm{~h}$. The suspension was thawed on ice and subjected to 5-10 passages through a 21 gauge needle to disrupt cells. Homogenate was transferred to $2 \mathrm{~mL}$ tubes and centrifuged at $18.000 \mathrm{x} g$ for 30 min at $4^{\circ} \mathrm{C}$. Supernatant was aspirated and pellet was suspended in PBSCM buffer. Protein concentration was determined by using the Pierce BCA Protein Assay. Membranes were used directly or stored at $-80^{\circ} \mathrm{C}$ until use.

For competition binding experiments ( $\mathrm{IC}_{50}$ determinations), $1-20 \mu \mathrm{g}$ membrane protein and was incubated with $0.2 \mathrm{nM}\left[{ }^{125} \mathrm{I}\right] \mathrm{RTI}-55$ in the presence of no or increasing concentrations of inhibitor in 96-well plates and total volume was adjusted to $150 \mu \mathrm{L}$ per well with PBSCM. Binding was allowed to equilibrate for $2 \mathrm{~h}$ on ice. Membranes were transferred to 96-well glass fiber filter plates (Unifilter C; PerkinElmer) preincubated with $0.1 \%$ polyethyleneimine by using a Packard Bell cell harvester and washed four times with water. Nonspecific binding was determined in parallel at membranes from non-transfected COS-7 cells. Filter plates were dried and soaked in MicroScint- 0 followed by counting of plates in a Packard TopCounter. For saturation binding studies (determination of $\mathrm{K}_{\mathrm{d}}$ ), increasing concentrations of $\left[{ }^{125}\right.$ I]RTI-55 diluted 1:15 with unlabelled RTI-55 was combined with 1 $20 \mu \mathrm{g}$ membrane in 96-well plates and total volume adjusted to $150 \mu \mathrm{L}$ per well with PBSCM.

Binding was allowed to equilibrate for $2 \mathrm{~h}$ on ice. Termination of the binding assay and quantification of the amount of accumulated radioligand was done by the same protocol as for competition binding experiments. Binding assays were carried out in duplicate and repeated at least three times.

Xenopus laevis oocyte expression and electrophysiology. hSERT mRNA was transcribed from the pGEM-HE Xenopus expression vector as previously described using the mMESSAGE mMACHINE T7 mRNA-capping kit (Ambion). ${ }^{2}$ Defoliated stage V-VI oocytes were prepared as described previously. ${ }^{3}$ All procedures involving Xenopus laevis were performed in adherence to a protocol approved by the Danish Veterinary and Food Administration (license 2014-15-0201-00031). Oocytes were injected with $15 \mathrm{ng}$ hSERT mRNA and incubated at $18{ }^{\circ} \mathrm{C}$ in standard Barth's solution which contained in mM: $88 \mathrm{NaCl}, 1 \mathrm{KCl}, 0.41 \mathrm{CaCl}_{2}, 2.4 \mathrm{NaHCO}_{3}, 0.33 \mathrm{Ca}\left(\mathrm{NO}_{3}\right)_{2}, 0.82 \mathrm{MgSO}_{4}$, and 5 Tris (pH 7.4) supplemented with $50 \mu \mathrm{g} / \mathrm{mL}$ gentamicin. Two-electrode voltage-clamp recordings were performed 4-7 days after injection by placing oocytes in a superfusion chamber and impaling with electrodes filled with $3 \mathrm{M} \mathrm{KCl}$. Oocytes were under continuous superfusion with Frog Ringer's solution (in mM: $115 \mathrm{NaCl}, 2 \mathrm{KCl}, 5$ HEPES, and $1.8 \mathrm{BaCl}_{2} ; \mathrm{pH} 7.6$ ) for the duration of recording. Oocytes were clamped at $-80 \mathrm{mV}$ and recordings were performed using an OC-725C amplifier (Warner Instruments Corp.) and a CED 1401 analogue-digital converter (Cambridge Electronic Design). Ligands were dissolved at $10 \mu \mathrm{M}$ in Ringer's solution and applied using an automated programmable perfusion system (Bioscience Tools). 


\section{Computational methods.}

Protein preparation. The hSERT model was prepared as described previously. ${ }^{4}$ Briefly, the sequence of hSERT was aligned to dDAT and modeled in an outward-open conformation using Modeller version 9.12. ${ }^{5}$ The resulting structure was subjected to optimization of a ten-residue segment in EL2 using the ultra-extended loop optimization algorithm in Prime. ${ }^{6}$ The model was prepared using the Protein Preparation Wizard in the Schrödinger Suite 2012. Glu508 and Asp524 were modeled in their protonated states, His 143 was modeled as the $\varepsilon$-tautomer, and a cysteine bridge was included between Cys200 and Cys209. Two sodium ions and one chloride ion were manually inserted, and the model was then minimized with distance restraints between each ion and their coordinating residues. The model was validated by its ability to reproduce the known binding mode of 5HT in the S1 site. ${ }^{7}$ Ligand preparation. The three bivalent PEG4-linked compounds $(\mathbf{2}, \mathbf{6}, \mathbf{1 0})$ were modeled and used in subsequent docking calculations. The protonation state of each compound was predicted by Epik version 2.3 (Schrödinger Suite 2012, Schrödinger, LLC, New York) ${ }^{8,9}$ and the tertiary amino groups in each compound were modeled as positively charged. Each compound was built in Maestro version 9.3 and minimized using MacroModel version 9.6 (Schrödinger Suite 2012, Schrödinger, LLC, New York) followed by a conformational search using the OPLS-2005 force field ${ }^{10}$ and implicit water in MacroModel version 9.6 (Schrödinger Suite 2012, Schrödinger, LLC, New York). Each step in the conformational search was followed by a 5000 step minimization or until the minimization reached convergence using a conjugate gradient algorithm. The resulting lowest-energy conformation of each compound was used in the IFD calculations.

Induced-fit docking. Each of the three bivalent PEG4-linked compounds $(\mathbf{2}, \mathbf{6}, \mathbf{1 0})$ was docked into hSERT using the IFD protocol. ${ }^{11}$ This protocol accounts for protein flexibility as well as ligand flexibility in three steps: first the ligands are docked into the protein using a reduced van der Waal $(\mathrm{vdW})$ potential effectively making both ligand and receptor atoms smaller. Secondly, the protein side chains within $5 \AA$ of the docked ligand are optimized around the ligand in order to accommodate ligand binding, and lastly, the ligand is re-docked using the full vdW potential. This protocol employs Glide version 5.8 and Prime version 3.1 (Schrödinger Suite 2012, Schrödinger, LLC, New York). The binding site was collectively defined as the centroid between Asp98 and Ile172 from the S1 site, and Glu493 from the S2 site. The first Glide docking was set up to retrieve a maximum of 200 poses with energies within $50 \mathrm{kcal} / \mathrm{mol}$ of the lowest-scoring pose, while the second docking was set up to retrieve a maximum of 100 poses that were within $30 \mathrm{kcal} / \mathrm{mol}$ of the lowest-energy pose. The final scoring was achieved using the XP protocol, and all other settings were default. The resulting poses were clustered using the Conformer Cluster script available through the Schrödinger website (https://www.schrodinger.com/scriptcenter?category_gui=1109). The script clusters poses based on their conformation, as evaluated by the root mean square deviation, and an average linking method using the hierarchical clustering algorithm from Canvas version 1.5 (Schrödinger Suite 2012, Schrödinger, LLC, New York).

Data analysis. The experimental data from uptake and binding assays were analyzed by nonlinear regression analysis using GraphPad Prism version 6.0 (GraphPad Software, La Jolla, CA, USA). Inhibitor $\mathrm{K}_{\mathrm{i}}$ values were calcuated from the $\mathrm{IC}_{50}$ values using the equation $\mathrm{K}_{\mathrm{i}}=\mathrm{IC}_{50} /\left(1+\left([\mathrm{L}] / \mathrm{K}_{\mathrm{x}}\right)\right)$, where $\mathrm{x}$ is either " $\mathrm{m}$ " or " $\mathrm{d}$ " whether it is an uptake or binding assay, respectively, and [L] is the concentration of ${ }^{3} \mathrm{H}$ - or ${ }^{125} \mathrm{I}$-labelled radioligand. All data are expressed as mean \pm SEM from at least three independent experiments carried out in triplicate (uptake assays) or duplicate (binding assays). Statistical analyses were performed by Student's $t$-test or one-way analysis of variance and values of $\mathrm{p}<0.05$ were considered statistically significant. 


\section{Supporting references}

1. Stuhr-Hansen, N.; Andersen, J.; Thygesen, M. B.; Strømgaard, K. (2016) Synthesis of symmetrical and non-symmetrical bivalent neurotransmitter ligands. ChemistrySelect 3, 407-413.

2. Söderhielm, P. C.; Andersen, J.; Munro, L.; Nielsen, A. T.; Kristensen, A. S. (2015) Substrate and inhibitor-specific conformational changes in the human serotonin transporter revealed by voltageclamp fluorometry. Mol. Pharmacol. 88, 676-88.

3. Poulsen, M. H.; Lucas, S.; Bach, T. B.; Barslund, A. F.; Wenzler, C.; Jensen, C. B.; Kristensen, A. S.; Strømgaard, K. (2013) Structure-activity relationship studies of argiotoxins: selective and potent inhibitors of ionotropic glutamate receptors. J. Med. Chem. 56, 1171-81.

4. $\quad$ Andersen, J.; Ladefoged, L. K.; Wang, D.; Kristensen, T. N.; Bang-Andersen, B.; Kristensen, A. S.; Schiøtt, B.; Strømgaard, K. (2015) Binding of the multimodal antidepressant drug vortioxetine to the human serotonin transporter. ACS Chem. Neurosci. 6, 1892-900.

5. Sali, A.; Blundell, T. L. (1993) Comparative protein modelling by satisfaction of spatial restraints. $J$. Mol. Biol. 234, 779-815.

6. Zhao, S.; Zhu, K.; Li, J.; Friesner, R. A. (2011) Progress in super long loop prediction. Proteins 79, 2920-35.

7. $\quad$ Celik, L.; Sinning, S.; Severinsen, K.; Hansen, C. G.; Møller, M. S.; Bols, M.; Wiborg, O.; Schiøtt, B. (2008) Binding of serotonin to the human serotonin transporter. Molecular modeling and experimental validation. J. Am. Chem. Soc. 130, 3853-65.

8. Greenwood, J. R.; Calkins, D.; Sullivan, A. P.; Shelley, J. C. (2010) Towards the comprehensive, rapid, and accurate prediction of the favorable tautomeric states of drug-like molecules in aqueous solution. $J$. Comput. Aided Mol. Des. 24, 591-604.

9. Shelley, J. C.; Cholleti, A.; Frye, L. L.; Greenwood, J. R.; Timlin, M. R.; Uchimaya, M. (2007) Epik: a software program for $\mathrm{pK}_{\mathrm{a}}$ prediction and protonation state generation for drug-like molecules. $J$. Comput. Aided Mol. Des. 21, 681-91.

10. Kaminski, G. A.; Friesner, R. A.; Tirado-Rives, J.; Jørgensen, A. M. (2001) Evaluation and reparametrization of the OPLS-AA force field for proteins via comparison with accurate quantum chemcial calculations on peptides. J. Phys. Chem. B 105, 6474-6487.

11. Sherman, W.; Day, T.; Jacobson, M. P.; Friesner, R. A.; Farid, R. (2006) Novel procedure for modeling ligand/receptor induced fit effects. J. Med. Chem. 49, 534-53. 